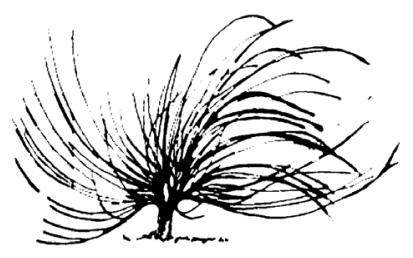

\title{
¿Qué sabemos que se enseña en filosofía?: El currículo oculto de una formación filosófica
}

Omar J. Ureña Soto ${ }^{1}$

Universidad Nacional

Costa Rica

omar.urena.soto@hotmail.com

Adrián Mata Calderón ${ }^{2}$

Universidad Nacional

Costa Rica

amaca08@hotmail.com

“Any Pérez: (...) ¿Cerraría la Escuela de Filosofia de la Universidad de Costa Rica?

José Miguel Corrales: Téngalo usted por seguro, que si necesitamos dinero para investigación, urgente, es mejor

cerrar filosofia y que los muchachos se preparen en la ciencia y en la investigación que es lo que le va dar grandes méritos a Costa Rica (...)”.

Debate Presidencial, CONARE, $2014^{3}$

\section{¿Un currículo bajo cuestionamiento?}

Preguntas como las siguientes: ¿para qué sirve la filosofía? ¿Por qué estudiar filosofía? ¿Cuál es el producto social que aporta un filósofo? ¿Hacia dónde va la disciplina? ¿Qué hace un filósofo?, encuentran respuestas absolutamente disímiles en razón del interlocutor. Ahora bien, dentro de la lógica del desarrollo, propia de los gobiernos

1 Estudiante maestría en Pedagogía Universitaria Educología, CIDE, UNA

2 Estudiante licenciatura en Pedagogía con énfasis en Didáctica Educología, CIDE, UNA

3 https://www.youtube.com/watch?v=7Lb4PG-XhXM A la literalidad a partir del minuto 1:03:20 hrs. 
neoliberales de América Latina y de la óptica imperialista, o desde las llamadas carreras técnicas o productivas, la respuesta es unívoca: la filosofía es una disciplina anacrónica, en el tanto no responde a los intereses del mercado.

Hasta aquí se ha expuesto una problemática contextual, lugar común que podría llevarnos a debatir sobre el objeto de estudio de la filosofía, su elemento teleológico, entre otras discusiones cuya deliberación, además de ser digna de otro tipo de trabajo, no ha encontrado consenso ni aún al estar en el patíbulo (caso de la filosofía en las sociedades actuales).

Aquí se pretende exponer, desde nuestra experiencia como graduados del Bachillerato en Filosofía y en Enseñanza de la Filosofía por la Universidad Nacional, como antiguos miembros de la Asociación de Estudiantes de Filosofía y como estudiantes de Pedagogía, el currículo oculto del Plan de Estudios del Bachillerato en Filosofía de la UNA.

¿De qué se habla cuando sale a relucir el tema sobre el currículo en educación? ¿Qué es? ¿Cómo funciona? Si bien el concepto fue utilizado con cierto sentido en su instauración, tanto su significado como sus implicaciones se han modificado y siguen transformándose con el paso del tiempo; sobre todo a partir de la segunda década del siglo XX. Diversos investigadores son citados constantemente como referencias obligatorias (Bobbit, Tyler, Schwab, Stenhouse, etc.), pues sus aportes y discusiones sobre la problemática curricular sentaron las bases para los posteriores desarrollos conceptuales. A grandes rasgos, y como señala Molina (2015):

La educación [...] constituye un proceso que pretende llevar a cabo las intencionalidades de un país en torno a la formación de sus ciudadanos. Este proceso se concentra en el nivel de las intencionalidades, mientras el currículo es la propuesta concreta que incluye una serie de experiencias previstas para hacer realidad o materializar esas intencionalidades. (p. 17)

No obstante, y de manera muy acertada, la educadora australiana Shirley Grundy (1998) indica lo siguiente al inicio del primer capítulo de su libro titulado Producto o praxis del currículum: 
A menudo se escribe y habla del "curriculum" en un sentido idealista, como si se tratase de una "idea" (eidos) perfecta de $\mathrm{cu}$ rriculum en relación con la cual todos los curricula concretos fueran imitaciones más o menos imperfectas. [...] Sin embargo, el curriculum no es un concepto, sino una construcción cultural. (p. 19)

El currículo constituye, teniendo en cuenta lo anterior, "una forma de organizar un conjunto de prácticas educativas humanas" (Grundy, 1998, pp. 19-20). Es una unificación de elementos, contemplando la teoría que sustenta la praxis pedagógica, los fines de la educación y la concreción del proceso de enseñanza-aprendizaje. Por otra parte, pensar en el currículo como una construcción cultural nos remite inmediatamente a las siguientes interrogantes: ¿cómo se construye? ¿con qué contenidos? Y más importante aún: ¿quiénes participan de ese proceso?

Es preciso que en la investigación sobre el curriculum que se desarrolla en las aulas se utilicen metodologías más etnográficas y participantes, junto a marcos de análisis más amplios, en los que se tengan en cuenta las interrelaciones entre el sistema educativo y lo que acontece en otras esferas de la sociedad, de esta manera podremos captar más fácilmente las conexiones entre el curriculum explícito y oculto de la institución escolar y las producciones económicas, culturales y políticas. (Torres, 2005, p. 10)

Lo mencionado por Jurjo Torres (2005) abre las puertas a la discusión sobre los distintos tipos de currículos, los cuales no son excluyentes en su puesta en práctica dentro de la dinámica educativa. Existen, cuando menos, cuatro tipos de currículo: explícito u oficial, paralelo o alternativo, emergente o espontáneo y oculto o invisible.

Como su nombre sugiere, el currículo explícito es aquel que la institución educativa pone en práctica de manera oficial; es decir, responde a los intereses de quienes ostentan el poder de elección respecto a los contenidos y didácticas del sistema educativo.

El currículo paralelo corresponde a las prácticas educativas que se llevan al espacio de aula, de manera consciente y alternativa, aunque no estén contenidas dentro del currículo oficial. Cabe mencionar también que no es azaroso, sino más bien planificado y controlado. 
Por su parte, el currículo emergente se presenta de modo espontaneo, surge cuando se toman en cuenta las eventualidades de la clase. Responde directamente a los intereses del estudiantado, siendo el rol de las y los docentes ser mediadores/as y facilitadores/as de información referente a los contenidos propuestos en clase. Es precisamente por esta naturaleza que se requiere un conocimiento crítico por parte del profesorado en temas relacionados con la experiencia cotidiana y los problemas que pueden surgir dentro del contexto de un grupo de estudiantes.

Finalmente, el currículo oculto, que corresponde al tema de investigación de este ensayo, es aquel que contiene las actitudes y valores, los estereotipos y los prejuicios, así como los ejercicios de resistencia y lucha, que inciden en las relaciones dentro de la práctica educativa y que se "manifiestan" de modo invisible. Es inconsciente, lo que significa que sus premisas están enraizadas y naturalizadas dentro del imaginario social de los y las docentes.

Es necesario, en este punto, señalar que existe ya un esfuerzo crítico, no en un sentido propiamente pedagógico, ni con pretensiones de apuntar a lo que surge consecuencia del currículo oficial; sino más bien se trata de un trabajo que pone de manifiesto carencias e inconsistencias del plan de estudios del Bachillerato de Filosofía de la Universidad Nacional. Al respecto, el trabajo de Daniela Soto Acuña (2012), una investigación profundamente valiosa a la cual recurriremos ineludiblemente, en el desarrollo del trabajo, cuando se aborden los euro-androcentrismos de los que, en apariencia, goza el currículo oficial.

La pregunta ¿qué se aprende en filosofía? 4 Será la que guiará las líneas siguientes. Lo primero para abordar la pregunta será aclarar en qué lugar se circunscribe la interrogante: La Universidad Nacional, Escuela de Filosofía y su respectiva historia, que nos decantó a la formación filosófica actual que reciben los y las estudiantes en el bachillerato (Sección A).

Posteriormente, será imperativo analizar, según su composición actual, los debates que ya han surgido de la aplicación del plan de estudios y, a su vez, la percepción del estudiantado, académicos

4 Se podría señalar que, en vista del carácter subjetivo con el que se ha manejado la problematización, la pregunta correcta debería ser: ¿qué aprendimos en filosofía? Sin embargo, hemos recogido más opiniones que la nuestra, censando a estudiantes del Bachillerato en Filosofía. Esto se pondrá de manifiesto más adelante. 
y administrativos sobre este. Se analizará cuáles contenidos fueron transmitidos por la simple forma del plan de estudios y cuáles son las implicaciones que tiene este en la realidad, según su contenido ideológico; será esto lo que se nominará en este trabajo currículo oculto (Sección B).

\section{Sección A: UNA filosofía que husmea en otras Unidades Académicas}

Tenía razón Adorno (1998) en Educación para la emancipación, cuando apuntó que no se trataba, en la educación, de solo transmitir conocimiento, o de formar desde afuera, sino que debíamos entender que esta debía ser emancipadora; es decir, la educación, entendida como un proceso, debería lograr la autonomía de los sujetos que se ven envueltos en él. Ahora bien, Adorno no planteó una defensa de la emancipación por la emancipación; más bien, precisaba que dicha categoría debía atender a la comprensión de dos problemas: la organización del mundo en el que vivimos y de la ideología dominante, solo entonces podríamos hablar de un para qué en la Educación. En este trabajo se pretende develar ambos aspectos.

¿A partir de cuándo? ¿Por qué? ¿Cuál ideología motivó la organización de una Escuela de Filosofía en la Universidad Nacional? ¿Qué objetivos perseguía el currículo oficial del Bachillerato en Filosofía tal cual lo entendemos ahora?

Para hablar de Filosofía en Costa Rica, en términos de su conformación institucional, parece necesario señalar a un individuo: Constantino Láscaris-Comneno Micolaw 5 . Fue este español, llegado a Costa Rica a finales de la década de los años cincuenta (1957), quien fuera promotor del modelo de Estudios Generales en la Universidad de Costa Rica (UCR), y a su vez en la Universidad Nacional (Mora, 1993), y cuyos aportes institucionales se dieron principalmente en la UCR.

Ahora bien, lo que aquí se pretende desarrollar prescindirá de Láscaris-Comneno en gran medida. No se trata de una cuestión antojadiza, sino que el período que se pretende retratar tuvo que seguir sin este, ya que murió al iniciar el proceso de institucionalización de la

5 Es sostenido por muchos que el avance de la filosofía, en el campo institucional, principalmente, se debió a Láscaris-Comneno. Basta ver las obras de Arnoldo Mora, Costa Rica en el siglo XX, Historia del pensamiento costarricense, o bien, la destacable labor que ha realizado el Profesor Alexander Jiménez. 
filosofía en la UNA (1979). Sin embargo, los precedentes marcados en su labor en la UCR lo vuelven relevante, como se verá más adelante.

La Universidad Nacional nació sin filosofía, nació sin Departamento, nació sin Escuela. Su vinculación a esta fue, directamente, en relación con otra casa de estudios, la UCR (Núñez, 1974). Los proyectos que tuvieron que ver con la intervención filosófica en una Universidad que aún se configuraba a la vista y paciencia de la realidad que la invocó, fueron impulsados, porque no existía otro medio, por profesionales de la Universidad de Costa Rica y extranjeros.

¿Por qué la UNA, centro de estudios de educación superior, decidió obviar en sus currículos a la filosofía? Los motivos se podrían enumerar en modo apertus, pero en el fondo se trató de una cuestión ideológica. ¿En qué sentido?

El argumento que resonó para, en aquel entonces, negarle un espacio a la filosofía, fue el de la duplicidad, ya existía la carrera en la UCR; si creamos una nueva Universidad, ¿para qué duplicar carreras, áreas de conocimiento?

Fueron varios los debates llevados a cabo sobre la posibilidad de formación, en la Universidad Nacional, de un Departamento centrado ya únicamente en la filosofía como disciplina. Se encontraron diversas reticencias a esto.

No fue sino hasta la sesión N. 14 del Consejo Universitario, artículo 5, fechada el 5 de junio de 1975, que se decidió crear el Departamento de Filosofía, con varios detractores y una interesante discusión con argumentos que, al parecer, no pasan de moda ${ }^{6}$. Por ejemplo, en la citada acta se lee en palabras del entonces miembro del Consejo Universitario, tiempo después Rector de la Universidad, el Dr. Araya Pochet:

El Dr. Araya Pochet pregunta si queda claro que iese Departamento de Filosofía será nada más una unidad coordinadora de los estudios de filosofía? Lo cual es imprescindible. ¿No se abrirá en el futuro la carrera de filósofo? Lo que preocupa es que si se puede seguir una política de duplicidades innecesarias, como se consideraría en estos momentos la Carrera de Filósofo en Costa Rica, cuando se tiene una Unidad Académica que ha tenido muchos años y mucha especialidad en la Universidad de Costa Rica

6 Al día de hoy la discusión, en el nivel nacional, es la misma. Hay exhaustivos ejemplos de esto. 
y la Universidad Nacional tiene la necesidad histórica de responder a una serie de Carreras que el país está requiriendo. (p. 10)

Argumento que sin duda opera bajo la idea de que la filosofía, en el fondo, no es una carrera útil, no para la Universidad Necesaria ${ }^{7}$. El temor a destinar recursos a la filosofía surge una y otra vez; basta ver, páginas más adelante:

La Lic. Naranjo Coto está de acuerdo con la creación del Departamento como Unidad Académica de Coordinación entre las distintas filosofías que se dan en las Unidades. Comparte así mismo las inquietudes del Dr. Araya Pochet en cuanto a la carrera de filósofo. Pero ¿cuál sería el costo de ese Departamento? (p. 11)

Después de que se aclaran las dudas y se afirma, por parte del Rector, para aquellos años el sacerdote Benjamín Núñez, que el presupuesto no se verá afectado más que por el aumento que recibirá alguno de los profesores que ya labora dando clases de filosofía en la Universidad, por asumir el cargo de Director de Departamento, y que además no se creará una carrera de filosofía ${ }^{8}$, sino que el Departamento tendrá solamente la función de coordinar todo lo referente a la disciplina, se procede a crearlo. Es claro entonces que el Departamento surge para coordinar. No más, no menos.

Luego de aquella histórica sesión en la que el Departamento de Filosofía, después de complicadas labores de parto, vio la luz; el Dr. Láscaris muere poco tiempo después (1979). Sin embargo, al finalizar la década de los setenta, se puso en marcha un proyecto en la Universidad Nacional que se nominó "Proyecto Láscaris", que acogió como estandarte de la Filosofía, como imagen, al profesor español (Saxe, 2014).

7 Es así como se ha concebido históricamente a la Universidad Nacional. En la Administración Figueres Ferrer (1970-1974), se contó con el expresidente de la República, el señor Óscar Arias Sánchez, quien para aquella Administración fungió como Ministro de Planificación, como uno de los ideólogos en la configuración de tal Universidad Necesaria. La idea de una Universidad que pretende satisfacer necesidades se encaminó (y así ha sido hasta la fecha) bajo una lógica del desarrollo, propia de los gobiernos de América Latina de aquella época. Dicha percepción invocaba una Universidad que, bajo la óptica del neoliberalismo, pretendía y pretende ser eficiente y competitiva en razón del avance social (relato hegeliano del desarrollo, propio de la Modernidad). Buscó integrar a personas de las zonas rurales del país para incluirles al mercado.

8 ¿Por qué era esto tan importante de aclarar y algo para ser categóricos en la discusión? 
Dicho proyecto pretendía publicar en diversos tomos toda la obra del filósofo y, además, darle el nombre del español a la biblioteca del Centro de Estudios Generales de la Universidad, entre otras labores, que ayudaron a la difusión filosófica y al mantenimiento de la memoria histórica.

El proyecto añadió impulso a una disciplina que, desde su incursión institucional, se enfrentó al cuestionamiento, a la interpelación económica y a una lógica del desarrollo que parecía encarar su existencia en las utilidades.

Láscaris además, antes de su muerte, propuso la creación de un Departamento en la Universidad Nacional, nominado entonces Instituto de Teoría e Historia de la Ciencia y la Tecnología. La finalidad de tal Instituto era la de abordar las recientes y crecientes innovaciones tecnológicas que acontecían en un mundo con un diseño heraclíteo; mundo que la filosofía encontraba la obligación de interpelar (Saxe, 2014). El Instituto fue dirigido, mientras existió, por el Dr. Roberto Murillo, quien también fue una figura emblemática en el proceso de la institucionalización de la filosofía en Costa Rica. Al desaparecer el Instituto, todo lo asignado a este pasó a ser asumido por el Departamento de Filosofía de la Universidad, que era a su vez, administrativamente, el órgano competente para tal labor.

Dicho Instituto, a pesar de su corta vida, generó diversos aportes al proceso de institucionalización de la filosofía: el impulso para diseñar una carrera adscrita al Departamento de Filosofía (futuro bachillerato en la Enseñanza de la Ciencia y la Tecnología), así como la producción intelectual de los Cuadernos Prometeo (que se mantienen hasta hoy día).

Los Cuadernos Prometeo pretendían, en sus anaqueles y nacientes de tinta, explorar las problemáticas propias del avance de la tecnología en el mundo y el rol de la filosofía en este avance y en las dinámicas de cuestionamiento y respuestas a estas.

Lo anterior, aunado a la creación de la Revista Praxis $^{9}$ en el año 1975, por parte del Departamento de Filosofía, en la que se profundizó sobre temas con incidencia teórico-social, se trató de publicaciones que

9 Nos atreveríamos a afirmar que el nombre no es gratuito, principalmente por el contenido que caracterizó a la Revista en aquellos años (principalmente influido por el materialismo dialéctico en boga en América Latina, opositor del liberalismo de la época) y además, por las ansias de un grupo de intelectuales dispuestos a hacer avanzar la filosofía en la Universidad, principalmente en el ámbito institucional; dispuestos a dar la lucha contra las prácticas de la Universidad Necesaria, o más bien, dispuestos a reafirmar que la filosofía es necesaria. 
pretendieron precisamente ser eso: praxis. Se procuró demostrar que la filosofía no solo consistía en una abstracción perdida en preguntas irresolubles, sino que también era una disciplina de un carácter práctico, que podía intentar, con resultados, transformar la realidad.

Los temas de publicaciones fueron varios y confrontativos, por ejemplo, el Profesor Carlos Molina y su artículo de 1979 en la Revista Praxis, titulado Notas sobre el concepto de la Práctica de la Filosofía en Costa Rica. El Materialismo Dialético, pone en cuestión, en gran medida, los problemas que hemos retratado hasta el momento: "A algunos que ejercemos la filosofía en la Universidad Nacional de Costa Rica, se nos presenta en forma dramática la necesidad de saber suficientemente qué es la filosofía, pues nos urge aclarar qué estamos haciendo o qué deberíamos hacer" (p. 5).

Es claro que, en la coyuntura abordada hasta ahora, existía una necesidad constante de justificar a la filosofía en sus condiciones institucionales y en los requerimientos de avance.

La filosofía en la UNA encontró cuestionamientos ya no solo desde la propia Administración de la Universidad ${ }^{10}$, sino ahora desde la UCR. Desde la Escuela de Filosofía de la UCR surgieron interrogantes respecto a la creación del Departamento de Filosofía de la UNA, principalmente fundados en una aparente duplicidad de funciones.

La pregunta de fondo era: ¿si ya existe una Unidad Académica en el país que se encargue de la filosofía como disciplina, para qué otra? En términos más específicos: ¿cuál es la motivación de descentralizar la filosofía de la UCR? ${ }^{11}$

Sobre esto acontecieron férreas riñas. Basta observar la participación del Exdirector del Departamento, y también Exdirector de la Escuela de Filosofía de la UNA, el Dr. Eduardo Saxe Fernández, en una Mesa Redonda en el año 1983, intitulada "Función del Departamento de Filosofía de la UNA en la Sociedad Costarricense":

Es cierto que hasta ahora ha resultado difícil establecer programas y formas de trabajo conjuntos entre la Escuela de la UCR y

10 Después del paso de Benjamín Núñez como Rector de la Universidad, entre otros, asumió la Rectoría el Dr. Araya Pochet, quien, como vimos más arriba, no se encontraba precisamente a favor del avance de la filosofía en la Universidad, o al menos tenía sus reservas con el tema. Se trataba de reservas ideológicas, en función de la Universidad Necesaria.

11 En términos generales se veía, según lo exponen diversos académicos de la UNA que abordaremos pronto, como un insulto para los colegas de la UCR. 
nuestro Departamento. Estas dificultades se reducen en el fondo al sustentamiento, por parte de dos grupos de filósofos, de posiciones filosóficas contrarias: nosotros intentando producir la filosofía que necesita el pueblo costarricense, para su proceso de emancipación histórica; en la UCR, en términos generales, simplemente transmitiendo y cultivando una filosofía idealista que ha servido para apoyar a aquellos que han adquirido granjerías a costa del sufrimiento y sudor populares. En la UNA pues, hacemos filosofía que extrae lo universal de lo costarricense, y viceversa. Y en la UCR, simplemente una filosofía desarraigada, "universalista", en un sentido peyorativo. (p. 23)

Ante la polémica que medió el desarrollo de la filosofía en la UNA, así como las constantes interpelaciones administrativas y disciplinarias, surge una pregunta: ¿qué implicaba coordinar para un Departamento en Filosofía? ¿Qué funciones tenía el Departamento de Filosofía de la UNA? ¿Difería realmente de la Unidad Académica de la UCR? Más allá de lo que podamos responder o pensar, el problema posee un enfoque erróneo. No se trataba de cuáles labores eran distintas, o de si una Unidad Académica transformaba la realidad y la otra no, o indistintamente de las diferencias epistemológicas ${ }^{12}$; el verdadero problema era: ¿qué incidencia se podría lograr desde la academia en la realidad costarricense a través desde los respectivos locus de enunciación? Ya que, de una u otra forma, para el contexto, eso era lo que posibilitaba la existencia institucional de la filosofía.

Ahora bien, tratándose de un Departamento que surge para coordinar lo referente a la filosofía en la Universidad, se encontraba inevitablemente en expansión. Ya para mediados de los años 80 tenía a su haber la administración de la Revista Praxis, la edición de los Cuadernos Prometeo y una carrera adjunta (Bachillerato en la Enseñanza de la Ciencia y Tecnología), además de la participación en diversos proyectos de extensión de la Universidad. Lo anterior consta en un interesante informe de labores del Departamento de Filosofía de la Universidad

12 Sin demeritar la discusión, la cual, sin duda, aportó muchísimo a los intelectuales de la época y ayudó a fortalecer las bases epistemológicas, los nortes (¿y sures?) académicos de estos y de las Unidades Académicas; sin embargo, lo fundamental, según el abordaje presentado, es que a su vez, consciente o inconscientemente, favoreció al proceso de avance institucional de la filosofía en Costa Rica. 
Nacional anunciado por el Dr. Alexis Ramírez, director del Departamento para el año 1980.

¿Impartía el Departamento de Filosofía, antes de la formación de su propia carrera adjunta, cursos en la Universidad? Efectivamente. Una de las funciones asignadas al Departamento de Filosofía, si bien recordamos (al no poseer carrera propia), era la de coordinar todo lo referente a la filosofía en la Universidad. Eso implicaba a los cursos del Centro de Estudios Generales relacionados con filosofía (la mayoría para aquellos años) y a su vez una gran cantidad de cursos de servicio ${ }^{13}$ que prestaban a otras Unidades Académicas.

Rodrigo Quesada Vargas, quien fue de los pocos que apoyó e impulsó la creación del Departamento de Filosofía en aquel debate del Consejo Universitario de la Universidad (1975), mencionó respecto de los cursos de servicio, ante la posibilidad de creación del Departamento: "En este momento los profesores que entrarían en el Departamento están dando los cursos de filosofía, de servicio, pero desperdigados en las diferentes Facultades" (p. 12).

Lo anterior refleja que se consideró a la filosofía, históricamente en su proceso de institucionalización, como una disciplina accesoria, no autosuficiente, sino en función de otra, instrumentalizada al servicio de otras disciplinas.

La cantidad de cursos de servicio impartidos por el Departamento de Filosofía era vasta. Para 1980 se puntualiza sólo para un curso en Humanidades intitulado "Fundamentos de Filosofía" un total de diecinueve grupos, y ofertados para toda la Universidad un total de sesenta y ocho cursos (Ramírez, 1980). Es decir, el Departamento de Filosofía, si bien no tenía un foco curricular definido (una carrera propiamente en filosofía), tenía una gran cantidad de docentes husmeando por toda la Universidad e incidiendo en la formación y producción intelectual de gran parte de esta ${ }^{14}$.

13 Los cursos de servicio, por lo general, reciben este nombre en razón de que una Unidad Académica de la Universidad los solicita a otra, o bien porque se encuentran a disposición de toda la población universitaria (solicitados por la Universidad a una Unidad Académica específica). Lo importante a destacar es que los cursos de servicio no son cursos de incidencia directa (sino más bien transversal o indirecta) en la formación o currículo oficial; sin embargo, aparecen como necesarios y parte del currículo oficial (se apela, en lo común, a un tema de interdisciplinariedad).

14 Llama poderosamente la atención que los cursos impartidos para las Escuela de Danza y Teatro en las áreas de "Historia del Teatro" e "Historia de la Danza", así como los relacionados a "Estética" o "Teoría Artística", eran impartidos por docentes del Departamento. 
La creación de una carrera propia por parte del Departamento de Filosofía, nominada Bachillerato en la Enseñanza de la Ciencia y la Tecnología, tuvo implicaciones interesantes en el proceso de institucionalización que venimos precisando. ¿Cuál era el fundamento de esta carrera? ¿Por qué un Departamento de Filosofía encargado de una carrera en ciencia y tecnología? Cuando Constantino Láscaris propuso esta idea, que se materializó, como vimos, bajo la dirección de Roberto Murillo, fue profundamente innovadora (Saxe, 2014). El surgimiento de la computación, la informática, así como el desarrollo tecnificado (cada vez más complejo) de la mecanización en la producción e industrialización necesitaban no solo explicación, sino de aplicación en relación con un mundo que, para aquellos años, se percibía cada vez más globalizado.

Como es bien sabido, la informática, la computación y sus incidencias tuvieron como fundamento teórico, en gran medida, los estudios de la filosofía analítica y de la filosofía del lenguaje; esfuerzos que se vieron materializados de alguna forma con Alan Turing, entre otros pensadores. Esto inspiró el surgimiento del primer plan de estudios a cargo del Departamento de Filosofía.

Esta carrera, al tenor de las condiciones materiales, se concretó en la realidad formando, entre otras labores, a profesores que impartieran los cursos del Departamento de Educación Técnica en la Sección de Educación Tecnológica del Ministerio de Educación Pública, dentro de las que destacaron, en un inicio, las asignaturas de Educación para el Hogar, Artes Industriales y Enseñanza de la Informática ${ }^{15}$.

El profesor Max Freund Carvajal, quien era ya para finales de los años ochenta un especialista en el área de la lógica (con estudios en el extranjero), en unión con otros colaboradores, se dieron a la tarea de fundamentar y modificar el plan de estudios para que en su núcleo poseyera un énfasis en pensamiento simbólico y lógica moderna ${ }^{16}$. Esto tuvo como consecuencia la formulación y creación de un nuevo plan de estudios, el cual se concretó para el año 1991 y se nominó Bachillerato en la Enseñanza de la Computación y la Informática.

15 Sobre esto ver, entre otras cosas, el programa del MEP del año 2000 referente a Educación para el Hogar.

16 Esto, a su vez, se podría considerar como un antecedente importante de la formación de una Escuela de Informática en la Universidad Nacional. Es interesante destacar que tanto el plan de Enseñanza en la Ciencia y la Tecnología, así como el nuevo plan que se diseñó, contaban con una cantidad importante de cursos del área de la lógica. 
Si bien es cierto Láscaris-Comneno fue visionario, la institucionalidad le desplazó este mérito a la filosofía, trasladando para el año 1995 el bachillerato, recientemente creado, a la Escuela de Informática de la Universidad. Lo anterior tuvo como consecuencia que, nuevamente, el Departamento reanudara su labor de disciplina accesoria, al únicamente ofrecer cursos de servicio a las otras Unidades Académicas (incluida la Escuela de Informática).

El Departamento se garantizaba su existencia, sin un plan de estudios propio, en razón de la gran cantidad de cursos que ofertaba. Sin embargo, las posibilidades de desmantelamiento y desplazamiento podían ser inminentes; existía, otra vez, la necesidad de justificar a la filosofía en la Universidad.

Consecuencia de los más de veinte años que tenía el Departamento de existir, su personal docente se encontraba, en su mayoría, especializado en el nivel de posgrado. Fue en razón de lo anterior que se propuso la idea de formular un currículo, por parte del Departamento, que ofertara el grado de doctorado; es decir, un programa de estudios de posgrado, el cual ya para finales de los años noventa era una realidad. Fue así como se ofertó el "Doctorado en Estudios Latinoamericanos con énfasis en Pensamiento latinoamericano", que se mantiene aún vigente.

Si bien el programa doctoral habilitaba tiempos de trabajo a profesores e incidencia por parte del Departamento en la realidad nacional y universitaria, existía, así como sucedió con la Escuela de Informática, un despojo de áreas, contenidos y cursos para el Departamento. Las otras Unidades Académicas necesitaban cada vez menos de la filosofía.

La filosofía en diálogo con otras disciplinas (enfoque interdisciplinario), como se concibió en un inicio (al únicamente ofertar cursos de servicio) será el eje posterior de la organización de un plan de estudios de grado: el "Bachillerato en Filosofía".

Este plan de estudios se concibió bajo la premisa de que, efectivamente, la filosofía debe proponer, en vista de la especialización, la posibilidad de problematizar desde conocimientos y áreas específicas, ya que el filosofar por el filosofar (la mera abstracción por la mera abstracción, la pregunta no especializada) perdía terreno en la realidad.

Al gozar de una historia distinta a la de la Universidad de Costa Rica, el Departamento de Filosofía de la Universidad Nacional siempre encontró la necesidad de justificar su funcionamiento. Consecuencia de sus condiciones y de los cuestionamientos, que no fueron escasos, su 
labor (en principio administrativa) debía ser propositiva e innovadora; si no encontraría un destino fúnebre. De ahí que se pensara en un plan de estudios de grado cuya finalidad, a pesar de llamarse "Bachillerato en Filosofía", no fuera formar especialistas en filosofia, sino la de brindar herramientas filosóficas para la problematización en otras áreas del pensamiento. Es decir, era un plan de estudios que pretendía prestar a profesionales formados, o en formación, de otras disciplinas, herramientas de interlocución con sus respectivas áreas de conocimiento; a fin de cuentas, el ideal de la interdisciplinariedad.

Siendo así, el plan de estudios del Bachillerato en Filosofía contó, desde su primera aparición, con un requisito de graduación hasta antes nunca visto en la historia de las universidades en Costa Rica: aportar un total de cuarenta créditos de otra disciplina; cerca de año y medio aprobado (tres ciclos, en las modalidades de universidad pública) en otra carrera. Esto era cerca de la obtención de un diplomado (si era el caso). La formulación del plan de estudios fue, sin duda, innovadora; e incluso se podría catalogar como brillante debido a la coyuntura (¡qué decir de la tradición del servicio!) que vivía el Departamento.

¿Qué sucedía con la duplicidad que se achacó respecto a la carrera ofertada en la UCR? Al respecto, en la fundamentación del plan enviada a Consejo Nacional de Rectores (CONARE) ${ }^{17}$ se exponía lo siguiente:

La carrera más afín propuesta es el Bachillerato y la Licenciatura en Filosofía de la Universidad de Costa Rica. Sin embargo, según la Universidad Nacional, existen diferencias notables entre dicha carrera y la propuesta. Como se estableció en la sección relacionada con el plan de estudios, hay veinte créditos dedicados específicamente a la filosofía de un área del saber o actividad humana. La carrera propuesta se enfoca más al planteamiento de problemas y soluciones filosóficas a diferentes áreas del saber y actividades humanas, intentando alcanzar de esta manera una gran interdisciplinariedad entre la filosofía por una parte, y las ciencias naturales por otra parte. (Consejo Nacional de Rectores, 1998, p. 7)

17 Órgano nacional encargado, en el nivel nacional, de la aprobación de carreras en las universidades. 
El currículo contenía en un inicio un total de 136 créditos repartidos de la siguiente forma:

- $\quad$ Estudios Generales (doce créditos)

- Idioma Instrumental (ocho créditos)

- Cursos obligatorios de Filosofía (cincuenta y seis créditos)

- Cursos optativos de un área específica de la Filosofía (veinte créditos)

- Cursos no filosóficos en el área de especialidad (cuarenta créditos)

Estos créditos se circunscribían en tres áreas específicas: de la filosofía, histórica y de especialidad. Todo lo dicho se pretendía abordar a través de tres ejes:

- $\quad$ Eje de problemas fundamentales

- $\quad$ Eje exegético de autores

- $\quad$ Eje de metareflexión

La idea que se generó en el Departamento era clara. En el primer eje se pretendía que los y las discentes comprendieran los problemas filosóficos fundamentales en sus diversas fases históricas, así como las alternativas de solución para propiciar una formación crítica. El segundo eje pretendería desarrollar las interpretaciones de la obra de un autor que ha marcado un hito en la historia de la filosofía y, por último, el eje restante propiciaría que el o la discente cursara asignaturas que le permitieran generar la capacidad de identificar problemas filosóficos que surgen, específicamente, en sus áreas de profesionalización. Lo anterior, por supuesto, en suma, con los $\mathbf{4 0}$ créditos que trajera consigo desde otra disciplina, o posteriormente aportara quien cumpliera los requisitos para ingresar a la Universidad, era lo necesario para poder graduarse de bachiller en Filosofía de la UNA.

El plan de estudios del Bachillerato en Filosofía tenía un matiz más y era la generación de un énfasis del graduando: los cuarenta créditos que aportara quien cursaba el plan definirían el énfasis de su bachillerato; es decir, si una persona aportaba esos cuarenta créditos en sociología, su 
título sería emitido como "Bachiller en Filosofía con énfasis en Ciencias Sociales", y así según el área en la que aportara los créditos ${ }^{18}$.

Siendo así, se escogieron unos autores (Platón, Aristóteles, Kant y Hegel), se delimitaron unos períodos históricos (Antigüedad, Edad Media, Modernidad y Contemporaneidad), y se desarrollaron cursos de problematización disciplinar (Filosofía de las Ciencias Naturales, de las Ciencias Sociales, del Arte y la Estética, de la Ética y la Bioética, de la Lógica, de la Política) a lo que se aunaron los cursos de metareflexión (que serían aquellos cursos que se deberían cursar, -optativos -, que deberían estar en una intrínseca relación con los 40 créditos que aportaba el o la ingresada al bachillerato).

Era claro que el plan de estudios pretendía reformular lo que históricamente se había entendido por filosofía; era un esfuerzo de contextualización y de volverla útil. La formulación de este plan de estudios se realizó cuando la dirección del Departamento de Filosofía se encontraba a cargo de la Dra. Ana Rodríguez Allen (especialista en ética y bioética). La comisión que elaboró el plan de estudios se encontraba conformada por la directora, el Dr. Max Freund Carvajal (profesor del Departamento) y el asesor curricular Carlos Retana (Soto, 2012).

El plan de estudios inició su puesta en práctica para el año 2000. Desde entonces ha sufrido diversas transformaciones y variantes ${ }^{19}$, siendo la última para el año 2014. Los cambios han sido más de contenido que estructurales; la estructura sigue siendo, esencialmente, la misma.

La Bach. Daniela Soto Acuña (2012) realizó un análisis en el que encontraba algunas deficiencias y contradicciones en el plan de estudios vigente. Soto señala, de forma atinada, tres defectos que pondremos a discusión de inmediato:

- $\quad$ El plan de estudios es eurocéntrico.

- El plan de estudios realiza una exclusión a la mujer en sus contenidos.

- La estructura actual del plan de estudios es contradictoria a los fines que representa.

18 Se contemplaron, en un inicio, los siguientes énfasis: artes y letras, ciencias sociales, ciencias naturales y ciencias de la salud. Estas áreas abarcaban gran parte de las disciplinas que eran objeto de estudios universitarios.

19 Empezando porque desde el 3 de febrero de 2006 el Departamento de Filosofía se convierte en Escuela de Filosofía. 
Si se analiza el plan de estudios actual, correspondiente al año 2014, lleva razón Soto en todo lo que plantea. Los autores que se contemplan en el plan son en su mayoría, y hasta hace poco en su totalidad, europeos y hombres; sin embargo, la ahora Escuela de Filosofía de la Universidad Nacional, en su actual administración ${ }^{20}$, se encuentra en la ejecución de esfuerzos para paliar estas deficiencias a través de la inclusión de ejes transversales de género en su plan de estudios, en conjunción con el Instituto de Estudios de la Mujer (IEM). Lo anterior de la mano de la realización de congresos, conferencias nacionales e internacionales, así como el impulso de publicaciones referentes a la filosofía y pensamientos latinoamericanos, distintas del enfoque europeo. Ahora bien, en lo referente a la modificación del plan de estudios esto no es palpable.

Por último, respecto a las contradicciones que presenta dicho plan, es imperativo acotar que las modificaciones que se le han realizado desde el año 2000, como atinadamente señala Soto, no van en armonía con su diseño inicial; lo anterior se puede constatar al echar un vistazo a todos los planes de estudios y sus respectivas modificaciones en el tiempo, lo que le permitió a Soto (2012) concluir que:

Inicialmente más de la mitad de la carrera se centraba en el eje de metareflexión, debido a la pretensión de ser complementaria o instrumento para profundizar en otras áreas del saber. Y sólo 36 créditos se centraban en problemas fundamentales y al exegético de autores, que al eje de metareflexión, el cual ha sido reducido a la mitad en la carga crediticia original; es decir, los primeros dos ejes han tenido un aumento de 20 créditos, los cuales le han sido quitados al tercer eje.

\section{La carrera se convierte así paulatinamente en una carrera de} Filosofía occidental autónoma y principal, no previendo su complementariedad, más bien, eliminando dicha naturaleza. ${ }^{21}$

Lo anterior nos da la oportunidad de concluir que una carrera cuya base es la interdisciplinariedad ha eliminado poco a poco, con el transcurso del tiempo, elementos que la vinculan con tal finalidad. Se

20 Bajo la dirección del M. Sc. Manuel Ortega Álvarez y el Lic. Andrés Gallardo.

21 La negrita no es del original. 
trata de que al comparar los planes de estudio del año 2000 y el vigente, hay una eliminación paulatina de los cursos que buscan darle el contenido interdisciplinar y cada vez se crean más cursos de historia de la filosofía en su reemplazo.

El tema de los cuarenta créditos responde, sin lugar a dudas, a un resabio de la historia que pesa sobre la institucionalización de la filosofía en la Universidad Nacional. La idea de una filosofía que dialoga, que problematiza y que está al servicio de las otras disciplinas es la que se encuentra detrás de esta forma curricular (que respondió a necesidades específicas y a condiciones materiales coyunturales).

Hasta ahora todo lo dicho brinda un panorama general de las bondades y algunas de las críticas que se han evidenciado y que facilitan comprender la naturaleza del plan de estudios del Bachillerato en Filosofía de la Universidad Nacional. Sin embargo, ¿cómo funciona en la praxis el diálogo de la filosofía con las otras disciplinas?

\section{Sección B: La pedagogía como área de metareflexión: una reflexión no realizada}

"El educador que aliena la ignorancia, se mantiene en posiciones fijas, invariables. Será siempre el que sabe, en tanto los educandos serán siempre los que no saben. La rigidez de estas posiciones niega a la educación y al conocimiento como procesos de búsqueda".

Paulo Freire, 2006.

Analizados, de forma general, los contenidos, ejes temáticos y aspiraciones del plan de estudios, nos basta para exponer con detalle algunas de sus limitaciones, y preguntarnos por el lugar que ocupa la pedagogía. ¿Por qué se vuelve importante hablar de pedagogía en relación con lo que se ha expuesto hasta ahora?

La Pedagogía, entendida en términos simples como una reflexión crítica sobre el fenómeno educativo, enfrenta un interminable esfuerzo de autocrítica, revisión y renovación constante. Esto, de forma general, debería darse por sentado al hablar de pedagogía universitaria, desde una visión deontológica; empero, apelando a la inmanencia, al 
campo de lo que es (ontológico), es tal vez el principal desafío (Giroux, 2011): el ejercicio, la existencia, re-creación de una Pedagogía Universitaria.

Como es bien sabido, la Universidad, así como la Escuela, es una institución paralítica (Salaman y Thompson, 1984), ya que depende de otras instituciones y centros de ejercicio del poder, no solo para moverse, sino para ser. Es evidente que dichas dinámicas de poder le restan autonomía a la Universidad ${ }^{22}$.

La Universidad, en su génesis medieval-escolástica, nos recuerda que es una ficción, cuya configuración y respuesta social depende de la coyuntura histórica en la que se encuentre. Su autonomía, su configuración administrativa, docente, estudiantil y la existencia de pedagogía ${ }^{23}$, responden a la ideología dominante (Torres, 1998).

Ahora bien, un plan de estudios y su puesta en práctica deberían ser objetos de la reflexión pedagógica, entendida en los términos que se han expuesto. En el caso particular del Bachillerato en Filosofía, los cuarenta créditos encuentran particular relevancia para un ejercicio de reflexión pedagógica.

Como hemos visto hasta ahora, estos cuarenta créditos encuentran sentido únicamente en razón de las áreas de metareflexión. ¿Cómo ha acontecido en la praxis el tema de los cuarenta créditos? ¿Ha existido un ejercicio de reflexión sobre estos? Lamentablemente no, cuando menos en el nivel institucional.

En la práctica acontecen, en el caso de los estudiantes del Bachillerato en Filosofía con los que tuvimos acercamiento durante y después de nuestra formación institucional, los siguientes hechos:

- La mayoría no cuenta con los cuarenta créditos al ingresar a la carrera de filosofía, por lo que debe, en el desarrollo de su formación en la UNA, para poder culminar sus estudios de grado, ingresar a otra carrera universitaria. El problema es que la Universidad no facilita los mecanismos, sino que quien ya se encuentra cursando el bachillerato podría quedar estancado (sin posibilidad

22 En la realidad nacional costarricense: ¿de qué autonomía se está hablando en las negociaciones con el Gobierno Central en el tema del presupuesto de la educación (FEES)? ¿Las vinculaciones con el Banco Mundial?

23 Son bien sabidos los matices que mediaron a las Universidades en sus orígenes, las luchas sociales que motivaron la concepción de la Universidad como autónoma, así como los cambios sobre quiénes imparten y reciben clases en esta a través del tiempo. 
de graduarse) hasta que no encuentre la posibilidad de acceder a otra carrera universitaria, lo que disminuye y limita considerablemente la promoción de estudiantes graduados en Filosofía por la UNA.

- $\quad$ No existe información clara que refiera a los estudiantes las implicaciones y posibilidades que tienen para cursar esos cuarentas créditos.

- $\quad$ No existe claridad, a priori, sobre los cursos o créditos que se le podrán reconocer a los estudiantes de filosofía en lo referente a los cuarenta créditos.

Pongamos en cuestión lo siguiente: ¿cuál es el lugar de la pedagogía en el Bachillerato en Filosofía? ¿Existe reflexión pedagógica respecto del Bachillerato en Filosofía? ¿Es considerada siquiera la pedagogía como un área de metareflexión?

La respuesta de la Dra. Ana Rodríguez Allen (Escuela de Filosofía Universidad Nacional, 2016, p. 9), quien, como vimos, colaboró en la confección del actual plan de estudios, es que la pedagogía no solo no es un área de metareflexión contemplada en el plan de estudios, sino que no podría serlo.

Las situaciones ontológicas que se presentan en la cotidianidad de los estudiantes del bachillerato se pueden esclarecer a través de la exposición de un ejemplo (entre varios). En el año 2016, en una Asamblea de la Escuela de Filosofía, realizada en los términos del Estatuto Orgánico de la Universidad ${ }^{24}$, se discutió sobre los cursos con contenido pedagógico. La pregunta sobre la que giró el debate fue la siguiente: ¿qué valor, para el plan de estudios de Filosofía, tienen los cursos con contenido pedagógico? ¿Se podrían reconocer estos como parte de los cuarenta créditos? ¿Es la pedagogía entonces un eje de metareflexión?

La discusión surgió en razón de la situación concreta de un estudiante: el ahora bachiller Juan Ureña. El señor Ureña se encontraba cursando, además del Bachillerato en Filosofía (cuyo componente filosófico había terminado, es decir, encontraba pendiente solo aportar los cuarenta créditos de otra disciplina para finalizar su plan de estudios), el Bachillerato en la Enseñanza de la Matemática.

24 En la que se contaba con representación del sector académico, sector administrativo y sector estudiantil de la Escuela de Filosofía. 
Ureña tenía más de 40 créditos aprobados en el Bachillerato en la Enseñanza de la Matemática; sin embargo, el Consejo de Escuela ${ }^{25}$ le había negado la posibilidad de graduarse con los créditos que aportaba ya que no formaban parte del eje de metareflexión. La situación, por tanto, en razón de una apelación a la resolución del Consejo de Escuela, fue elevada a la Asamblea de Escuela, para que en plenario decidiera al respecto.

En dicha Asamblea, que consta en actas de Asamblea de Escuela, a la que encontrábamos legitimación para asistir junto con otros y otras compañeras en calidad de representantes estudiantiles, se dilucidaron al menos tres interpretaciones en razón de la situación del estudiante:

1. La carrera de Enseñanza de la Matemática, al ser una carrera compartida en términos curriculares, encuentra un eje de metareflexión (la matemática) y un componente pedagógico (que no formaría parte de ningún eje de metareflexión); por lo que los únicos cursos que le posibilitarían graduarse al estudiante serían aquellos que sean referidos única y exclusivamente al eje de metareflexión, es decir, a la matemática. El estudiante no podría graduarse ese año. Posición defendida por la Dra. Ana Rodríguez Allen y otros académicos.

2. La carrera de Enseñanza de la Matemática, al ser una carrera compartida en términos curriculares, encuentra un eje de metareflexión (la matemática) y un componente pedagógico (también eje de metareflexión). Sin embargo, los únicos cursos que le posibilitarían graduarse al estudiante serían aquellos que sean referidos a solo uno de ambos ejes, ya que el título que se emitiría por parte de la Universidad está referido a un único énfasis; es decir, un solo título no podría otorgar a un discente dos énfasis (para el caso concreto ciencias exactas y ciencias sociales), por lo que no es posible contar la totalidad de los créditos aprobados, sino que el estudiante debería escoger uno. El estudiante no podría graduarse ese año. Posición defendida por el subdirector de la Escuela, el Lic. Andrés Gallardo.

3. Por último, la posición defendida por el director de la Escuela, el M. Sc. Manuel Ortega Álvarez, los y las Representantes

25 Órgano inferior, en la burocracia universitaria, respecto de la Asamblea de Escuela. 
Estudiantiles, el sector administrativo y dos académicos (la M. Sc. Diana Solano Villareal y el Dr. Ronald Casas), fue la de que la Asamblea de Escuela no debería realizar distinciones que no puntualiza la normativa, por lo que se debían aceptar los cuarenta créditos sin mayor distinción que la reglamentaria (lo referente a los problemas de la doble titulación en relación con el Bachillerato en la Enseñanza de la Filosofía); lo anterior implicaba que el énfasis del título sería emitido con el eje de metareflexión en el que el estudiante tuviera más créditos aprobados, reconociendo ambas disciplinas como ejes de metareflexión. El estudiante se podría graduar a la brevedad.

El debate aconteció, se produjo el contradictorio y al realizarse la votación del órgano entre tres argumentaciones absolutamente válidas, se concluyó darle la razón a la tercera posición expuesta y el estudiante Ureña se pudo graduar en el ciclo correspondiente. De no ser así, el estudiante, según las otras interpretaciones, hubiera tenido que cursar al menos dos años más para poder graduarse de Bachiller en Filosofía.

Esta es una situación que no es aislada en la dinámica de la Escuela de Filosofía y los cuarenta créditos. Estas y otras discusiones son producto de la estructura curricular, que pareciera bienaventurada, pero que administrativamente encuentra problemas serios de aplicación debido a su falta de claridad.

Ahora bien, llevaba razón la Dra. Rodríguez Allen en que, definitivamente, en la formulación del plan de estudios desde el 2000 a la actualidad, no se contempló a la pedagogía como un eje de metareflexión. El plan de estudios se encuentra abiertamente encaminado hacia la reflexión y problematización de áreas de corte técnico u tecnológico (basta ver el sentido de los ejes de metareflexión). Llevaba razón sin duda el Lic. Gallardo en que administrativamente existía una limitación (entre muchas otras, que no se consideraron al formular el plan de estudios), y es que se vuelve maniqueo: o es eje de metareflexión o no lo es. Es por esto último que no podrían comulgar más de dos ejes de metareflexión.

¿Cuál es el problema del no reconocimiento de la pedagogía en el plan de estudios? La imposibilidad de la valoración de la aplicabilidad no solo académica o curricular, sino en relación con la realidad administrativa, por parte del plan de estudios. Esto tiene consecuencias 
en la validación de determinados contenidos y la descalificación ideológica por parte de otros que administrativa o académicamente no se consideren importantes. Es decir, si la pedagogía no es un eje de metareflexión, no se puede saber a ciencia cierta en qué dirección se realizan las modificaciones curriculares, a quiénes afectan, o con qué intenciones se realizan. La pedagogía, cuando se creó el plan de estudios, ni siquiera fue considerada como una disciplina digna de interlocución, según expresó la Dra. Rodríguez Allen.

De entrada, el plan de estudios le niega la posibilidad o descalifica estas reflexiones al ser de índole pedagógicas, ya que no considera a la pedagogía como un eje sobre el que se pueda filosofar. Siendo así, la pedagogía diría que esto no es inocente (Torres, 2005). La elección de contenidos, el hecho de la preeminencia del eurocentrismo, la no sensibilización de género, el pensar una filosofía instrumentalizada (al servicio de otras disciplinas) ${ }^{26}$, brindan sin duda herramientas para hablar de un currículo oculto.

¿Cuál es el currículo oculto del que hablamos? Al finalizar el Bachillerato en Filosofía se comprende que, eminentemente, el desarrollo de las ideas y el pensamiento se da en Europa, que en su mayoría las mujeres han aportado poco o casi nada en la consecución de pretensiones filosóficas, que la filosofía es una disciplina paralítica; sin embargo, lo principal, y que necesariamente resulta importante señalar: que en el contexto de una Universidad que surge para el mercado se crea una filosofía y se reproduce hoy una carrera en filosofía para el mercado. Lo opuesto a esto no está en el papel, se ha tratado de organización y de luchas, para la visibilización de estos problemas.

Después de censar a un total de 60 estudiantes de la carrera del Bachillerato en Filosofía, entre los niveles de primero a cuarto, se dilucidaron los siguientes resultados:

- $\quad$ Más del $75 \%$ no tiene claro por qué debe cursar 40 créditos en otra carrera, o bien las razones que da no son las que justifican la existencia del currículo oficial.

- $\quad$ El $100 \%$ no sabe, específicamente, cuáles son los ejes de metareflexión del plan de estudios.

26 Ni siquiera existe un cuestionamiento serio sobre estas pretensiones. No existe una reflexión endógena por parte de la Escuela de Filosofía en lo referente a esto. 
- $\quad$ Más de un $60 \%$ no ha leído a ninguna autora en filosofía durante el cumplimiento de su plan de estudios.

¿Eso explica la baja promoción de la carrera de filosofía? ¿La deserción, o más bien expulsión (D'Antoni, 2008), de estudiantes respecto de ese programa de estudios?

Pareciera ser que el problema que enfrentamos está relacionado con la idea de interdisciplinariedad que inspiró este plan de estudios. No está mal la interdisciplinariedad, no es contra eso la queja, sino contra esa interdisciplinariedad que pretende dialogar con otros, sin dialogar primero sobre el diálogo; aquella "interdisciplinariedad" que no es interdisciplinariedad, porque nunca pretendió discutir sobre sus condiciones, posibilidades, sino que se dice interdisciplinaria en razón de que su partida es multidisciplinar. Eso no nos parece suficiente, pues asumir que se dialoga no es lo mismo que dialogar. Para hablar de diálogo entre disciplinas deben estar claras las condiciones materiales, de realidad, para que se dé el diálogo: quiénes, de cuáles formas y sobre qué se va a entablar el diálogo; algo así como la Utopía de la que nos habla Hinkelammert (1984). La incertidumbre administrativa, las relaciones de verticalidad (Unidad Académica - discentes) deben clarificarse.

En este punto, nos parece que el estudiantado, el profesorado, e incluso la Unidad Académica, se encuentran dando una lucha de resistencia contra las dinámicas universitarias y la incertidumbre de cómo aplicar el currículo vigente. Las reformas al plan de estudios que buscan reducir su componente (de pretensión) interdisciplinario, las políticas y vinculaciones de la Universidad con el mercado, la incerteza de académicos, administrativos y estudiantes sobre cómo opera y cómo operar el currículo son muestra de que hace falta realizar un análisis pedagógico (crítico) profundo. Para esto necesitamos parar con la endogamia filosófica. Hemos creado, con nuestras prácticas de resistencia (desde la filosofía), más confusión y desorden porque no logramos lidiar con el cambio coyuntural y con en el currículo dado que, entre otras cosas, le negamos desde el principio el diálogo a la pedagogía.

En el caso del Bachillerato en Filosofía debemos empezar a considerar los problemas de "¿qué enseño-aprendo y cómo lo hago?" 
como inherentemente pedagógicos, para establecer (a partir de definir el cómo) un diálogo con la filosofía. La práctica educativa no es inocente, y debemos clarificar los supuestos éticos, políticos, epistemológicos y estéticos bajo los que vamos a responder al problema de la educación en nuestra disciplina.

\section{A modo de cierre:}

En atención a las precisiones que se realizaron al inicio de este trabajo, sobre la posibilidad de un currículo -una educación- para la emancipación, es necesario que realicemos unas reflexiones finales.

Ante las dificultades que se ha evidenciado históricamente para justificar a la filosofía, como disciplina, en el nivel institucional, a causa del desarrollo del neoliberalismo -en razón de la creación de una Universidad para el mercado-, no se puede obviar la discusión sobre la ideología dominante y las instituciones que la soportan. En este contexto, una educación para la emancipación debe partir por reconocerse oprimida (Freire, 2006).

La misma Escuela de Filosofía, en su necesidad de legitimarse dentro de la dinámica institucional, no reconoce las prácticas de resistencia ante la burocracia, y reproduce prácticas de currículo oculto que no se presentan en oposición a la ideología dominante; dentro de ellas el no reconocimiento de una pedagogía crítica.

La posibilidad de que ambas disciplinas entablen un diálogo crítico brindaría no solo las herramientas para entender que la educación, en general, debe facilitar la inclusión de sus participantes, sino además para la construcción de un currículo paralelo que pretenda paliar las deficiencias -limitaciones en general-, así como los procesos sistémicos de exclusión (D'Antoni, 2008) que genera la burocracia; lo anterior, al menos, hasta que no exista una discusión seria sobre la necesidad de replantear el actual plan de estudios.

Bajo este escenario, la esperanza de apelar a una filosofía que pretenda la transformación de sus circunstancias y de construir un currículo para la liberación se perfila como una utopía que, consecuencia de las actuales condiciones materiales, se ve imposibilitada para Ser.

La obligación de plantear preguntas para la acción y de posibilitar el cambio para esta utopía recae, principalmente, sobre quienes ostenten la legitimidad burocrática. Son ellos y ellas quienes deben de fomentar 
la discusión, generar espacios de problematización sobre el currículo y a su vez promover la participación, abierta, para sus modificaciones, por parte de todos los sectores (académico, administrativo, estudiantil).

En conjunción con lo anterior, pareciera que se vuelve imprescindible modificar, en primera instancia, la lógica que le dio vida al plan de estudios:

La Dra. Ana Rodríguez (...) Agrega que, por otra parte, en el documento de CONARE, plan de estudios original nunca se pensó en esos 40 créditos en términos pedagógicos porque lo que se pensaba tenía más que ver con desarrollar cuadros académicos vinculados con la epistemología, la filosofía de las ciencias y la filosofía política. (Escuela de Filosofía Universidad Nacional, 2016, p. 9).

Dicha lógica debe apelar a la idea de una filosofía que dialoga; pero esta vez primero con las personas, luego con las disciplinas.

\section{Referencias}

Adorno, T. W. (1998). Educación para la emancipación. Madrid: Morata.

Consejo Nacional de Rectores. (1998). Dictamen sobre la propuesta de creación del Bachillerato en Filosofía. Oficina de Planificación de la Educación Superior. OPES 20/98.

Cordero, G., Ramírez, A., Saxe, E. (1983). Función del Departamento de Filosofia de la UNA en la sociedad costarricense. Recuperado de http://www.repositorio.una.ac.cr/bitstream/handle/11056/2356/recurso_454.pdf?sequence $=1$ \&isAllowed $=\mathrm{y}$

D'Antoni, M. (2008). Nuevas propuestas pedagógicas y el papel social del docente. Ensayos Pedagógicos, 4(1), 11-26.

Departamento de Filosofia, Universidad Nacional. (s. f.). Breves aclaraciones sobre el Plan de estudios de la carrera de Bachillerato en la Enseñanza de la Tecnología en ... (Según certificado de carácter técnico). Recuperado de http://www.repositorio.una.ac.cr/ bitstream/handle/11056/2174/recurso_272.pdf?sequence $=1$

Departamento de Filosofía, Universidad Nacional. (s. f.). Plan de estudios de la carrera de Bachillerato en la Enseñanza de la Tecnología con énfasis en... (Según certificado de carácter técnico). Recuperado de http://www.repositorio.una.ac.cr/bitstream/handle/11056/2394/recurso_491.pdf?sequence=1\&isAllowed=y 
Departamento de Filosofía de la Universidad Nacional (2000). Plan de Estudios del Bachillerato en Filosofia. Costa Rica: DFUNA.

Escuela de Filosofía, Universidad Nacional. (2012). Plan de estudios: Bachillerato en Filosofia. Costa Rica: EFUNA.

Escuela de Filosofía, Universidad Nacional (2014). Plan de estudios: Bachillerato en Filosofía. Costa Rica: EFUNA.

Escuela de Filosofía, Universidad Nacional. (2016). Acta N. ${ }^{\circ} 02-2016$, Unidad Académica. Costa Rica: EFUNA. (Sin publicar).

Freire, P. (2006). Pedagogía del oprimido. Buenos Aires: Siglo Veintiuno Editores.

Giroux, H. (2011). Teoría y resistencia en la educación. México, D. F.: Siglo XXI editores.

Grundy, S. (1991). Producto o praxis del currículum. Madrid: Morata. Hinkelammert, F. (1984). Crítica a la razón utópica. San José: DEI.

Molina, C. (1979). Notas sobre el concepto y la práctica de la filosofía en Costa Rica. En Praxis, enero-junio, N. ${ }^{\circ} 11-12$, pp. 5-14. Costa Rica: EUNA.

Molina, Z. (2015). Fundamentos del currículo. Costa Rica: EUNED.

Mora, A. (1993). Historia del pensamiento costarricense: Costa Rica: EUNED.

Núñez, B. (1974). Hacia la Universidad Necesaria. Recuperado de http://www.documentos.una.ac.cr/bitstream/handle/ unadocs/5972/hacia\%201a\%20Universidad\%20Necesaria. pdf? sequence $=1 \&$ isAllowed $=\mathrm{y}$

Ramírez, A. (1980). Departamento de Filosofia: Informe Anual de Labores 1980. Recuperado de http://www.repositorio.una.ac.cr/ bitstream/handle/11056/2240/recurso_339.pdf?sequence=1

Salaman, G. y Thompson, K. (1984). Control e ideología en las organizaciones. México: Fondo de Cultura Económica.

Saxe, E. (2014). Constantino Láscaris Comneno y el derecho humano a una memoria digna. Hoja Filosófica. ISSN: 2215-4051. Vol. 1. N. ${ }^{\circ} 35$. Diciembre. pp. 16-23.

Soto, D. (2012). Evaluación diagnóstica al Plan de Estudios de Bachillerato en Filosofía de la Universidad Nacional de Costa Rica. Escuela de Filosofía, UNA. Costa Rica: Sin publicar.

Torres, J. (2005). El curriculum oculto. España: Morata. 